\title{
Heart function disturbances in chronic kidney disease - echocardiographic indices
}

\author{
Beata Franczyk-Skóra ${ }^{1}$, Anna Gluba ${ }^{1,2}$, Robert Olszewski ${ }^{3}$, Maciej Banach²,4, Jacek Rysz ${ }^{1,2}$
}

\author{
1Department of Nephrology, Hypertension and Family Medicine, WAM University \\ Hospital of Lodz, Lodz, Poland \\ ${ }^{2}$ Healthy Aging Research Center, Medical University of Lodz, Lodz, Poland \\ ${ }^{3}$ Department of Cardiology and Internal Medicine, Military Medical Institute \\ of Warsaw, Warsaw, Poland \\ ${ }^{4}$ Department of Hypertension, Medical University of Lodz, Lodz, Poland
}

Submitted: 21 October 2014

Accepted: 1 December 2014

Arch Med Sci 2014; 10, 6: 1109-1116

DOI: 10.5114 /aoms.2014.47822

Copyright $\odot 2014$ Termedia \& Banach

\section{Abstract}

Introduction: In chronic kidney disease (CKD) patients left ventricular (LV) diastolic dysfunction occurs frequently and is associated with heart failure (HF) and higher mortality. Left ventricular systolic dysfunction is associated with coronary artery disease (CAD) and is a major determinant of prognosis. The aim of this study was to assess indices of LV diastolic dysfunction in CKD patients.

Material and methods: Study included 118 CKD patients. All patients underwent transthoracic echocardiography. Diastolic function based on $E$ and $A, E / A$ ratio and pulmonary vein flow velocities as well as EF\%, deceleration time, RA, LA volume were assessed. In dialysis patients examination was carried out before and after dialysis.

Results: In CKD patients the stage of renal failure was associated with the significant increase in LV mass $(268.0 \pm 47.6$ CKD I/II vs. $432.7 \pm 122.4$ CKD V/dialysis, $p<0.0001)$, systolic LV $(37.3 \pm 4.5$ vs. $51.2 \pm 8.9, p<0.0001)$ and diastolic LV (CKD I-II $44.7 \pm 4.1$ vs. CKD III $48.5 \pm 6.7$ vs. CKD IV $47.1 \pm 5.6 ; p=$ $0.004)$ dimensions and in the size of the LA $(40.4 \pm 2.0$ vs. $41.9 \pm 2.7$ vs. 42.3 \pm 3.2 vs. $44.8 \pm 3.1 ; p<0.0001)$. The increase the $E / E$ ' ratio between groups of patients ( $6.7 \pm 1.5$ vs. $8.9 \pm 2.4$ vs. $11.5 \pm 4.0$ vs. $13.5 \pm 5.0 ; p<0.0001)$ was seen in this study. The reduction in deceleration time (247.2 \pm 34.5 in CKD I/ II vs. $197.4 \pm 61.0$ in CKD IV, $p=0.0005$ ) along with the decrease in estimated glomerular filtration rate was also observed in this study.

Conclusions: Early identification of factors involved is necessary to prevent this devastating process. Many indexes of contractility are used and each of them has imperfections. It seems that TVI, E, E/A and E/E' are good instruments for the early detection of left ventricular hypertrophy and diastolic dysfunction.

Key words: heart failure, indices, diastolic dysfunction, chronic kidney disease.

\section{Introduction}

Chronic kidney disease (CKD), which prevalence is still growing worldwide, confers a higher risk of coronary artery disease (CAD), chronic heart failure (CHF) and/or death independently of conventional cardiovascular risk factors [1-4]. In CKD patients left ventricular (LV) diastolic dysfunction occurs frequently and is associated with heart failure (HF) and high-

\author{
Corresponding author: \\ Prof. Jacek Rysz \\ Department of Nephrology, \\ Hypertension and \\ Family Medicine \\ WAM University Hospital \\ of Lodz \\ 113 Zeromskiego St \\ 90-549 Lodz, Poland \\ E-mail: jacek.rysz@skwam. \\ lodz.pl
}


er mortality [5]. Other studies demonstrated that CKD severity was the most independent predictor of elevated LV filling pressure and could be responsible for impaired systolic and diastolic functions in predialysis CKD [6]. Left ventricular diastolic dysfunction is observed even in patients with early stages of chronic kidney dysfunction [7]. It was estimated that $15 \%$ of patients starting dialysis therapy have systolic dysfunction of the left ventricle while the prevalence of diastolic dysfunction at dialysis inception is much higher. Either systolic or diastolic dysfunction can lead to clinically evident congestive heart failure [8]. Left ventricular systolic dysfunction is often associated with severe $C A D$ and it is a major determinant of prognosis [9]. Left ventricular diastolic dysfunction in CKD patients is of complex nature. According to studies, it may be influenced by the increase in LV preload due to progression of CKD stage [10]. Also LV hypertrophy, CAD, microvascular abnormalities, interstitial fibrosis, altered fluid and electrolyte metabolism and neurohumoral alterations might contribute to the development of LV diastolic dysfunction in patients with CKD [11]. Over-activation of the rennin-angiotensin-aldosterone system (RAAS) might play an important role in the pathomechanism since even a mild CKD results in early cardiac fibrosis with mild LV diastolic impairment and preserved systolic function [12].

Therefore the aim of this study was to assess indices of LV echocardiographic indices in CKD patients including those on dialysis since the relationships between the echocardiographic parameters and renal function have not been fully examined.

\section{Material and methods}

This is a cross-sectional analysis of a population of CKD patients. The study included 118 patients from the Department of Nephrology, Hypertension and Family Medicine and the Dialysis Centre, WAM University Hospital of Lodz. The enrolled patients were categorized into the following CKD subgroups: stage II $(n=25)$, stage III $(n=30)$; stage IV $(n=28)$; and stage V/dialysis $(n=35)$. All patients signed informed consent form. Exclusion criteria were as follows: state after kidney transplantation, haemoglobin $<8 \mathrm{~g} / \mathrm{dl}$, active cancer or cancer diagnosed in the past, active hepatitis B or $C$, in an interview or repeatedly elevated blood levels of transaminases: alanine transaminase (ALT), aspartate transaminase (AST) alcoholism, malnutrition, HIV infection or other immune disorders, connective tissue diseases, therapy with immunosuppressive drugs, significant arrhythmias, state after implantation of heart pacemaker (CRT, ICD), history of venous thrombosis or pulmonary embolism, hyperthyroidism and hypothyroidism, he- modynamically significant heart disease, ejection fraction $(E F)<45 \%$, hypertrophic cardiomyopathy obesity, lack of consent to participate in the study. Criteria for inclusion in the study according to the criteria for the recognition KDOQI CKD and the recommendations of the ESC section of Echocardiography in 2009 on the recognition of diastolic dysfunction of the left ventricle.

All patients underwent transthoracic echocardiography (TTE) using Aloka ProSound Alpha camera 10. Measurements were made in the M-dimensional and two-dimensional 2D presentation. Flow parameters were evaluated using Doppler (continuous wave method - CW, pulse method- PM and tagged color method) and TDI. In the study, the following indices were assessed: size of the left atrium (LA), end-diastolic dimension of intraventricular septum (IVSd), left ventricle (LVIDd) and left ventricle posterior wall of the (PWd). The results of these measurements were used to evaluate left ventricular ejection fraction (EF\%) indicating LV systolic function and left ventricular mass index (LVMI). Characteristics of mitral inflow is the simplest and most commonly used technique for the assessment of diastolic function. The spectrum of mitral inflow was recorded using pulsed Doppler examination with Doppler gate placed at the end of mitral leaflets in apical 4-chamber view. Diastolic function was assessed by determining the velocities of early $(E)$ and late $(A)$ diastolic transmitral flow, the ratio E-to-A (E/A), deceleration time (DT), isovolumic relaxation time (IVRT) and pulmonary vein flow velocities. Indices of LV diastolic function were analysed depending on the severity of CKD in the study groups. On the basis of the aforementioned parameters three basic types of diastolic dysfunction: impaired relaxation (mild diastolic dysfunction with usually normal LV filling pressure at rest), pseudonormalization (moderate diastolic dysfunction with mildly or moderately elevated LV filling pressure) and restriction (severe diastolic dysfunction characterized by significantly elevated LV filling pressure) were distinguished. In dialysis patients the examination was carried out before and after dialysis. The severity of diastolic dysfunction is mirrored by the increase in LV filling pressure associated with a decrease in LV susceptibility due to the exacerbation of LV fibrosis and the increase in the stiffness of cardiomyocytes themselves. Results of echocardiographic examination were confirmed by tissue Doppler methods which could detect the subtle changes of diastolic function due to kidney dysfunction. Tissue Doppler was used since this method is less dependent on volume load in CKD patients. Echocardiographic examination was performed in accordance with the recommendations of the ESC section of Echocardiography of 2009. 


\section{Statistical analysis}

Data are presented as mean \pm standard deviation (SD). Shapiro-Wilk test was used to assess the normality of variables' distribution. Homogeneity of variance was checked with Levene's test. In case of a normal distribution and no differences in variances, the significance of differences between the two groups was assessed using paired sample $t$-test, and if at least one of the aforementioned criteria was not met Wilcoxon test was used.

\section{Results}

The mean age of patients with CKD II-IV was $63.97 \pm 12.5$ years and $67.78 \pm 12.0$ in dialysis group. There were more males in dialysis group than in other CKD group (68.57\% vs. $30.12 \%)$. In dialysis group hypertension ( $93.1 \%$ vs. $85.54 \%$ ) and lipid disorders (97.5\% vs. $87.95 \%)$ were more frequent in comparison to CKD stages II-IV. The frequency of treatment with $\beta$-blockers and Ca-channel blockers was similar in CKD II-IV and dialysis patients. Hemodialysis (HD) patient more rarely used angiotensin converting enzyme inhibitors (ACE-I) and angiotensin receptor blockers (ARBs). None of patients was treated with Ivabradin, Ranolazin or NO-donors. Diastolic dysfunction of right ventricle was also observed. Demographic characteristic is presented in Table I. Subjects with hypertension were more likely to have atrial fibrillation, increased diameter of LA, increased atrial volume before dialysis and decreased after it as well as reduced early diastolic velocity (E') and ejection fraction (data not shown).

The analysis of echocardiographic parameters showed that in CKD patients the stage of renal failure was associated with the significant increase in LV mass $(268.0 \pm 47.6$ CKD I/II vs. $287.8 \pm 70.3$ CKD III vs. $298.1 \pm 86.0$ CKD IV vs. $432.7 \pm 122.4$ CKD V/dialysis, $p<0.0001)$, IVSd (13.0 (12.0-14.2) in CKD I/II vs. 16.0 (15.0-17.0) in CKD V/dialysis, $p<$ $0.0001)$, IVSs (15.0 (14.7-17.0) in CKD I/II Vs. 18.0 (18.0-19.0) in CKD V/dialysis, systolic LV $(37.3 \pm 4.5$ vs. $51.2 \pm 8.9, p<0.0001)$ and diastolic LV (CKD I-II $44.7 \pm 4.1$ vs. CKD III $48.5 \pm 6.7$ vs. CKD IV $47.1 \pm 5.6$; $p=0.004)$ dimensions and in the size of the LA ( $40.4 \pm 2.0$ vs. $41.9 \pm 2.7$ vs. $42.3 \pm 3.2$ vs. $44.8 \pm 3.1$; $p<0.0001)$. Moreover, the increase in the $\mathrm{E} / \mathrm{E}$ ' ratio between groups of patients $(6.7 \pm 1.5$ vs. $8.9 \pm 2.4$ vs. $11.5 \pm 4.0$ vs. $13.5 \pm 5.0 ; p<0.0001)$ was seen in this study. The reduction in deceleration time (247.2 \pm 34.5 in CKD I/II vs. $225.6 \pm 43.2$ in CKD III vs. 197.4 \pm 61.0 in CKD IV, $p=0.0005)$ along with decreased estimated glomerular filtration rate (eGFR) was also observed in this study. The results of echocardiographic examination are summarized in Table II.

When echocardiographic indices were compared before and after the dialysis, only E/E' dif- fer significantly (13.5 \pm 5.0 before dialysis vs. 10.2 \pm 4.7 after dialysis; $p=0.002$ ). The rest of indices seems to remain unaffected by renal replacement treatment. Examination results are presented in Table III.

\section{Discussion}

The analysis of echocardiographic parameters in this study showed that in CKD patients increasing stage of renal failure was associated with the significant increase in both systolic and diastolic left ventricular dimensions and in the size of the left atrium. All these three parameters were greater in CKD stage V/dialysis and lowest in stage II. In more than half of the patients with formed arteriovenous fistula increased left ventricle end-diastolic dimension and worsening of diastolic function (shortening of deceleration time, $\mathrm{E}$ wave, the increase in E/A) were observed. Moreover, in agreement with Parfrey et al. [13] study, it has been observed that shortly after the dialysis session, a reduction in diastolic diameter of the LV and an increase in the thickness of the LV wall occur which is associated with volume depletion by ultrafiltration (data not shown). Also, in this study, the RV diameter was found to be much greater in CKD patients stage V/ dialysis $(29.9 \pm 2.9)$ than in stages II-IV. Additionally, steady growth of interventricular septal thickness in end diastole and systole as well as the increase of LV muscle mass were observed in CKD patients along with the rising CKD stage. Left ventricular muscle mass was over 1.5-times higher in dialysis patients that in CKD stage II subjects. According to Zoccali et al. [14] the increase in mass of $1 \mathrm{~g} / \mathrm{m} \mathrm{2.7/}$ month was associated with a $62 \%$ increase in the incident risk of fatal and non-fatal cardiovascular events in dialysis patients. They also suggested that changes in LV mass index represent a stronger predictor of mortality and cardiovascular complications than LV mass itself. Also Miyzato et al. [15] in

Table I. Demographic characteristic of study groups

\begin{tabular}{|lcc|}
\hline Parameter & Stage II-IV & V/Dialysis \\
\hline Sex, male (\%) & 30.12 & 68.57 \\
\hline Age [years] & $63.97 \pm 12.5$ & $67.78 \pm 12.0$ \\
\hline Hypertension (\%) & 85.54 & 93.1 \\
\hline Lipid disorders (\%) & 87.95 & 97.5 \\
\hline Diabetes (\%) & 28.92 & 25.6 \\
\hline ACE-I treatment (\%) & 31 & 49 \\
\hline$\beta$-blockers treatment (\%) & 42 & 45 \\
\hline ARBs treatment (\%) & 10 & 23 \\
\hline $\begin{array}{l}\text { Ca-channel blockers } \\
\text { treatment (\%) }\end{array}$ & 63 & 57 \\
\hline
\end{tabular}


Table II. Echocardiographic examination results

\begin{tabular}{|c|c|c|c|c|c|}
\hline Parameter & $\begin{array}{l}\text { II stage } \\
(n=25)\end{array}$ & $\begin{array}{l}\text { III stage } \\
(n=30)\end{array}$ & $\begin{array}{l}\text { IV stage } \\
(n=28)\end{array}$ & $\begin{array}{l}\text { V stage/dialysis } \\
\quad(n=35)\end{array}$ & Value of $p$ \\
\hline IVSd [mm] & $\begin{array}{c}13.0 \\
(12.0-14.2)^{9}\end{array}$ & $\begin{array}{c}13.0 \\
(12.0-13.2)^{9}\end{array}$ & $\begin{array}{c}13.0 \\
(12.0-14.7)^{9}\end{array}$ & $\begin{array}{c}16.0 \\
(15.0-17.0)\end{array}$ & $<0.0001$ \\
\hline IVSs [mm] & $\begin{array}{c}15.0 \\
(14.7-17.0)^{9}\end{array}$ & $\begin{array}{c}16.0 \\
(14.0-16.2)^{9}\end{array}$ & $\begin{array}{c}16.0 \\
(14.2-17.7)^{9}\end{array}$ & $\begin{array}{c}18.0 \\
(18.0-19.0)\end{array}$ & $<0.0001$ \\
\hline LV mass [g] & $268.0 \pm 47.6^{9}$ & $287.8 \pm 70.3^{9}$ & $298.1 \pm 86.0^{9}$ & $432.7 \pm 122.4$ & $<0.0001$ \\
\hline LV hypertrophy & 25 (100\%) & $30(100 \%)$ & $28(100 \%)$ & $30(100 \%)$ & NS \\
\hline LV systolic [mm] & $37.3 \pm 4.5^{9}$ & $41.0 \pm 6.2^{9}$ & $38.9 \pm 6.2^{9}$ & $51.2 \pm 8.9$ & $<0.0001$ \\
\hline LV diastolic [mm] & $44.7 \pm 4.1$ & $48.5 \pm 6.7^{8}$ & $47.1 \pm 5.6^{7}$ & $43.1 \pm 8.8$ & 0.004 \\
\hline LA diameter [mm] & $40.4 \pm 2.0^{5,9}$ & $41.9 \pm 2.7^{9}$ & $42.3 \pm 3.2$ & $44.8 \pm 3.1$ & $<0.0001$ \\
\hline RV diameter [mm] & $25.9 \pm 2.5^{9}$ & $26.8 \pm 4.0^{8}$ & $26.5 \pm 2.9^{9}$ & $29.9 \pm 2.9$ & $<0.0001$ \\
\hline$E / E^{\prime}$ & $6.7 \pm 1.5$ & $8.9 \pm 2.4^{1}$ & $11.5 \pm 4.0^{3,5}$ & $13.5 \pm 5.0$ & $<0.0001$ \\
\hline $\mathrm{E} / \mathrm{A}$ & $\begin{array}{c}0.80 \\
(0.75-0.90)\end{array}$ & $\begin{array}{c}0.80 \\
(0.70-0.90)^{8}\end{array}$ & $\begin{array}{c}0.80 \\
(0.60-1.20)\end{array}$ & $\begin{array}{c}0.96 \\
(0.81-1.31)\end{array}$ & 0.007 \\
\hline Deceleration time [ms] & $247.2 \pm 34.5^{6}$ & $225.6 \pm 43.2$ & $197.4 \pm 61.0^{7}$ & $269.0 \pm 135.6$ & 0.0005 \\
\hline $\begin{array}{l}\text { Diastolic disturbances } \\
\text { (relaxation disturbances) }\end{array}$ & $24(96.0 \%)$ & $29(96.7 \%)^{4}$ & $22(78.6 \%)^{8}$ & $19(54.29 \%)$ & 0.005 \\
\hline $\mathrm{EF} \%$ & $\begin{array}{c}56.0 \\
(55.0-60.0)^{1,4,9}\end{array}$ & $\begin{array}{c}50.0 \\
(50.0-55.0)^{9}\end{array}$ & $\begin{array}{c}50.0 \\
(50.0-55.0)^{9}\end{array}$ & $\begin{array}{c}45.0 \\
(40.0-50.0)\end{array}$ & $<0.0001$ \\
\hline
\end{tabular}

${ }^{1} p<0.05 ;{ }^{2} p<0.01 ;{ }^{3} p<0.001$ in comparison to stage $11 \mathrm{I} ;{ }^{4} p<0.05 ;{ }^{5} p<0.01 ;{ }^{6} p<0.001$ in comparison to stage $\mathrm{IV} ;{ }^{7} p<0.05 ;{ }^{8} p<0.01$; ${ }^{9} p<0.001$ in comparison to stage $V$.

Table III. Results of comparison of echocardiographic indices before and after dialysis

\begin{tabular}{|lccc|}
\hline Parameter & Pre-HD & Post-HD & Value of $p$ \\
\hline Left atrial volume VLA & $34.9 \pm 21.1$ & $34.4 \pm 20.9$ & NS \\
\hline Right atrial volume VRA & $31.4 \pm 19.6$ & $30.5 \pm 18.0$ & NS \\
\hline E' (LV) & $9.4 \pm 4.0$ & $9.0 \pm 4.7$ & NS \\
\hline E/E' (LV) & $13.5 \pm 5.0$ & $10.2 \pm 4.7$ & 0.002 \\
\hline E' $^{\prime}$ RV) & $12.5 \pm 5.4$ & $12.5 \pm 5.4$ & NS \\
\hline E/E' (RV) & $8.0 \pm 5.0$ & $8.3 \pm 6.0$ & NS \\
\hline E/A (LV) & $0.8(0.7-0.9)$ & $0.8(0.8-0.9)$ & NS \\
\hline SPAP & $27.0 \pm 17.2$ & $27.0 \pm 17.2$ & NS \\
\hline
\end{tabular}

their study of patients with chronic kidney disease noticed increased LV mass.

Systolic function of LV (EF parameter) remained within normal limits in all patients with a noticeable tendency to decrease with the decline in eGFR. EF\% was highest in CKD patients stage II, while lowest in stage V/dialysis. Although, this study population consisted of patients with preserved $E F$, there were significant differences between groups. Also Agarwal et al. [16] observed a progressive decline of LV ejection fraction with the increase in severity of renal failure. However, Hayashi et al. did not observe significant impairment of systolic function even in patients with severe CKD [17]. According to studies the occurrence of sys- tolic dysfunction of LV varies from $15 \%$ to $28 \%$ in patients on dialysis $[13,18]$.

In this study it has been analysed whether the progression of renal failure is associated with the $H F$, i.e. whether significant increase in left ventricular filling pressure, assessed on the basis of the ratio of transmitral early peak diastolic velocity to the early diastolic mitral annulus velocity (E/E') is observed. An E/E' ratio is particularly important in this assessment due to the fact that it reflects LV filling pressure which is the indicator of diastolic dysfunction [19, 20]. Numerous studies demonstrated that this ratio was the best non-invasive predictor of elevated LV filling pressure and final diastolic pressure among multiple 
echocardiographic indices $[21,22]$. The E/E' ratio $<8$ is considered to be normal, while the ratio $>15$ is considered to mirror the rise in the LV filling pressure $[23,24]$. Increasing E/E' ratio between groups of patients has been seen in this study. It was lowest in CKD patients stage II $(6.7 \pm 1.5)$, nearly two times higher in stage IV $(11.5 \pm 4.0)$ and even greater in dialysis patients before the procedure $(13.5 \pm 5.0)$. In dialysis patients this ratio was significantly lower after dialysis than before it $(p=0.02)$. Also Masugata et al. [10] demonstrated that the E/E' ratio increased with the decrease in eGFR and was significantly higher in CKD patients $(16.0 \pm 6.8)$ than in those with eGFR $>90 \mathrm{ml}$ $\mathrm{min} / 1.73 \mathrm{~m}^{2}$. Pecková et al. [25] observed the relationship between two echocardiographic parameters of LV diastolic function (E' and E/E' ratio) and reduced eGFR (stage I-III according to K/DOQI) in patients with type 2 diabetes without a prior history of cardiovascular events. Another study of patients with chronic kidney disease also revealed a significant deteriorated LV diastolic function parameters - E' and E/E' associated with lower eGFR [26]. Otsuka et al. [7] study confirmed that the severity of the kidney dysfunction parallels the rise of E/E' $(p<0.02)$. Results of a two-dimensional correlation analysis showed the relationship between eGFR and E/E' but not between eGFR and E'. However, according to the results of multivariate analysis both E/E' and E' were independent predictors of eGFR decline. Cluster analysis and ROC curves were used in order to explain this discrepancy and to assess the impact of E' on the relationship between E/E' and eGFR. These analyses showed that $E^{\prime}=7.1 \mathrm{~cm} / \mathrm{s}$ is the cut-off point and that the correlation between E/E' and eGFR was significant only for patients with E' $\leq 7.1 \mathrm{~cm} / \mathrm{s}$. This means that two different types of patients can be distinguished on the basis of the presence or absence of impaired LV relaxation. Cut-off point $\mathrm{E}^{\prime}=7.1 \mathrm{~cm} / \mathrm{s}$ corresponds to an age-related border value between the normal and impaired relaxation [27]. The results of another study suggest that abnormal LV relaxation is a factor underlying the relationship between non-invasive parameter E/E' and eGFR. This thesis is confirmed by the fact that there was no significant difference in eGFR between patients with and without abnormal relaxation. On the basis of these results, it can be hypothesized that the improvement of LV relaxation function may prevent or delay the development of heart disease and renal failure abnormalities in patients with uncomplicated type 2 diabetes mellitus [15]. The study by Barberato et al. [28] conducted on patients on dialysis revealed that diastolic velocities (early E' and late A') obtained in TDI analysis were 'relatively' independent of preload, did not significantly change after one session of hemodialysis and therefore E' parameter can be particularly useful for dialysis patients to differentiate between pseudonormalization and normal diastolic function profile [28]. The E/E' is of particular importance for the diagnosis of advanced diastolic dysfunction characterized by increased intracellular pressure. The study including 125 renal transplant patients showed that the E/E' $>15$ was an independent predictor of increased LV diastolic blood pressure (>15 mm Hg) and was associated with higher overall mortality in this group $[21,22]$.

The analysis of LV diastolic parameters on the basis of E/A ratio revealed that its values were similar in patients CKD stage II-IV which is consistent with adopted standards. However, this ratio was significantly higher in dialysis patients. Also in the study of Otsuka et al. [7] mitral E velocity was significantly lower in healthy subjects and CKD patients stage II-IV) ( $p<0.01-0.02)$ compared to stage $\mathrm{V}$, while mitral $A$ velocity was higher in stages $I I-V(p<0.01-0.04)$ than in healthy persons. Moreover, the ratio of mitral $E$ and $A$ velocities (E/A) was significantly higher in healthy patients $(p<0.02-0.05)$ compared to stages II-V. Otsuka et al. [7] suggested that A, E/A and E' could help to differentiate between healthy subjects and patients with early stage of CKD. In this study, E/A ratio did not differ before and after the dialysis.

The reduced deceleration time (DT) along with decreased eGFR was observed in this study. This time was shortest in CKD (excluding ESRD) patients stage IV and longest in II stage. Deceleration time in dialysis patients was much longer than that seen in CKD patients stage II-IV which is probably a result of lower volume overload due to ultrafiltration. Reduced DT as well as decreased values of E/A ratio are indicators of LV diastolic dysfunction and increased LV muscle stiffness. On the basis of obtained results it can be assumed that diastolic LV dysfunction occur in such an early stages of CKD as stage II. Also Otsuka et al. [7] in their study observed that DT was significantly longer in healthy subjects and CKD patients stage II $(p<0.01-0.02)$ than in CKD patients stage IV and $V$. Furthermore, in their study DT was significantly lower in stage $V(p<0.01)$ in comparison to stage IV. Diastolic dysfunction seen in CKD patients may precede hypertrophy, as well as the appearance of systolic LV dysfunction.

This study also revealed that the prevalence of left ventricular diastolic dysfunction in the form of relaxation disturbances was high - 96\% in patient with II CKD stage, $96,7 \%$ in III stage, $78,6 \%$ in IV stage and $54.29 \%$ in ESRD. Diastolic dysfunction develops in three phases: inversion of ratio of peak early to peak atrial velocity - curve, pseudo- 
normalization of E/A flow pattern and finally the development of a restrictive pattern with various degrees of early atrial flow velocity involvement $[19,29]$. In patients with long-lasting, severe CKD, LV diastolic dysfunction is more frequent in the form of pseudonormal and restrictive filling patterns of LV [28] and thus the percentage of patients with diastolic dysfunction in the form of relaxation disturbances observed in stage IV and $V$ is lower than in earlier stages. In $28.57 \%$ of patients with CKD stage $V$ diastolic dysfunction in the form of pseudonormalization was recognized and confirmed by TDI analysis. These patients had the most severe symptoms, significant impairment in exercise tolerance, shortness of breath at rest and periodic leg swelling. Restriction pattern was seen in $17.14 \%$ of these patients. According to other studies the incidence of diastolic LV dysfunction in CKD ranges from $50 \%$ to $65 \%$ including patients non-dialysed, those on dialysis and renal transplant recipients [30,31]. Moreover, some studies have shown that diastolic LV dysfunction precedes systolic function impairment and is responsible for approximately $30-40 \%$ of cases of heart failure [32]. The occurrence of diastolic dysfunction without significant impairment of systolic function was also observed in the study of Hayashi et al. [17]. On the basis of results obtained in this study it can be assumed that diastolic dysfunction occur in the early stages of CKD - even in stage 2 (eGFR $89-60 \mathrm{ml} / \mathrm{min} / 1.73 \mathrm{~m}^{2}$ ). Also Otsuka et al. study [7] demonstrated that LV diastolic dysfunction was observed even in patients with early stages of chronic kidney dysfunction and suggested that Doppler indices combined with conventional and tissue Doppler methods could detect the subtle changes of diastolic function due to kidney dysfunction. According to Masugata et al. [10] LV diastolic function may influence the increase in left ventricular preload as a result of CKD progression. Therefore, LV hypertrophy may be a better prognostic factor than the LV diastolic dysfunction in predicting low eGFR in patients with CKD. The increase in diastolic LV dimension observed in this study as well as very high prevalence of CKD-associated diastolic dysfunction may be an indicator of left ventricular volume overload which is due to over-hydration. This hypothesis is confirmed by the study of London [30] which shows that diastolic dysfunction is a common phenomenon in patients with advanced renal failure and end-stage renal disease due to hypertension and over-hydration. Furthermore, they concluded that renal impairment is associated with diastolic dysfunction regardless of the aetiology of renal failure [7]. Due to the fact that in non-dialysed patients with CKD it is sometimes difficult to control fluid volume (preload) and systemic blood pressure (load), advanced LV diastolic dysfunction observed in patients with kidney disease resulting from diabetic nephropathy suggests that diastolic heart failure in these patients can be induced by heart overload, even despite normal systolic function [33-36]. Hayashi et al. [17] suggested that in patients with advanced renal failure, factors other than LVH may cause diastolic dysfunction since patients with eccentric LVH and with severe renal failure but not patients with mild/moderate renal disease had significantly lower E' compared with controls. Moreover, patients with severe CKD showed more pronounced diastolic dysfunction than patients with mild/moderate CKD and controls though the prevalence of LVH did not differ between the groups [17]. Although LV filling abnormalities are frequently reported in patients with CKD, their prognostic significance is not known in this group of patients [37]. Nevertheless, it is common that left ventricular hypertrophy is a predictor of cardiovascular complications in patients with hypertension and CKD but the relationship between the echocardiographic parameters and renal function still are not fully explored [38, 39].

In CKD patients, left ventricular hypertrophy $(\mathrm{LVH})$ is a common finding and it is associated with an increased CVD-related mortality [17, 40]. In this study, LV hypertrophy was observed in all patients, but it was slightly more pronounced in patients with stage IV and V. The occurrence of LVH in this study was higher compared to that observed by Hayashi et al. [17] - 63\% in CKD patients stage I, II and III and 66\% in CKD stage IV-V. Studies indicates that the prevalence of LVH increases to $60-75 \%$ in patients beginning renal replacement therapy, and reaches $70-90 \%$ in patients on regular dialysis [41, 42]. According to Levin et al. [43] the prevalence and severity of LVH increases along with the progression of chronic kidney disease. At first, LVH is a physiological response to pressure and volume overload, but then when combined with anaemia and hyperparathyroidism it may turn into maladaptive LVH characterized by structural changes in the myocardium, such as collagen accumulation, fibrosis and calcification, which lead to systolic and diastolic dysfunction [17, 30, 44]. In patients on dialysis arteriovenous fistula reduces systemic vascular resistance [45] and increases stroke volume load on the left ventricle which may contributes to LVH and may results in LV systolic and diastolic dysfunction with time $[46,47]$. Also Rosello et al. [48] suggested that LVH contribute to diastolic LV dysfunction. According to Masugata et al. [10], LV hypertrophy may be superior to LV diastolic dysfunction in predicting low eGFR in patients with CKD using echocardiography. Hayashi et al. [17] suggested that LV geometry can affect systolic function since eccentric 
LVH has been described to be more frequently associated with systolic dysfunction than concentric LVH [17]. However, in this study systolic function remained within normal limits in all patients, even in those with end-stage renal disease.

The limitation of this study is the fact that the number of subjects was relatively small and all patients came from a single centre and thus there is a possibility of bias in the selection process. Greater study group is needed to produce a more accurate representation of the prevalence of diastolic dysfunction in CKD. Moreover, our study population consisted of CKD patients with normal LV EF [47-51].

In conclusion, in CKD patients maladaptive events leading to LVH, structural changes myocardium as well as diastolic dysfunction and even systolic failure occur frequently. Thus, early identification and treatment of factors involved in order to prevent this devastating process. Many indexes of contractility have been developed and each of them has imperfections. Now it seems that TVI and $E, E / A$ and $E / E$ ' parameters are good instruments for the early detection of LVH and diastolic dysfunction as they are important risk factors for cardiovascular morbidity and mortality in CKD.

\section{Acknowledgments}

Three authors are partially supported by The Healthy Aging Research Center project (REGPOT2012-2013-1, 7FP). No professional writer was involved in the preparation of this paper.

\section{References}

1. Pabst S, Hammerstingl C, Hundt F, et al. Pulmonary hypertension in patients with chronic kidney disease on dialysis and without dialysis: results of the PEPPER-Study. PLoS ONE 2012; 7: e35310.

2. Hatta T, Nishimura S, Nishimura T. Prognostic risk stratification of myocardial ischemia evaluated by gated myocardial perfusion SPECT in patients with chronic kidney disease. Eur J Nucl Med Mol Imaging 2009; 36: 1835-41.

3. Franczyk-Skóra B, Gluba A, Banach M, Rysz J. Treatment of non-ST-elevation myocardial infarction and ST-elevation myocardial infarction in patients with chronic kidney disease. Arch Med Sci 2013; 9: 1019-27.

4. Athyros VG, Katsiki N, Tziomalos K, et al.; GREACE Study Collaborative Group. Statins and cardiovascular outcomes in elderly and younger patients with coronary artery disease: a post hoc analysis of the GREACE study. Arch Med Sci 2013; 9: 418-26.

5. Kim MK, Kim B, Lee JY, et al. Tissue Doppler-derived E/E' ratio as a parameter for assessing diastolic heart failure and as a predictor of mortality in patients with chronic kidney disease. Korean J Intern Med 2013; 28: 35-44.

6. Hung MJ, Yang NI, Wu IW, et al. Three-dimensional echocardiographic assessment of left ventricular remodeling in predialysis chronic kidney disease patients. J Nephrol 2012; 25: 96-106.
7. Otsuka T, Suzuki M, Yoshikawa H, Sugi K. Left ventricular diastolic dysfunction in the early stage of chronic kidney disease. J Cardiol 2009; 54: 199-204.

8. Gluba-Brzózka A, Michalska-Kasiczak M, Franczyk-Skóra B, Nocun $M$, Banach $M$, Rysz J. Markers of increased cardiovascular risk in patients with chronic kidney disease. J Lipids Health Dis 2014; 13: 135.

9. Hida S, Chikamori T, Tanaka H, et al. Diagnostic value of left ventricular function after adenosine triphosphate loading and at rest in the detection of multi-vessel coronary artery disease using myocardial perfusion imaging. J Nucl Cardiol 2009; 16: 20-7.

10. Masugata H, Senda S, Goda F, et al. Echocardiographic assessment of the cardio-renal connection: is left ventricular hypertrophy or diastolic function more closely correlated with estimated glomerular filtration rate in patients with cardiovascular risk factors? Clin Exp Hypertens 2010; 32: 113-20.

11. Sato W, Kosaka T, Koyama T, et al. Impaired renal function is a major determinant of left ventricular diastolic dysfunction: assessment by stress myocardial perfusion imaging. Ann Nucl Med 2013; 27: 729-36.

12. Martin FL, McKie PM, Cataliotti A, et al. Experimental mild renal insufficiency mediates early cardiac apoptosis, fibrosis, and diastolic dysfunction: a kidney-heart connection. Am J Physiol Regul Integr Comp Physiol 2012; 302: R292-9.

13. Parfrey PS, Foley RN, Harnett JD, Kent GM, Murray D, Barre PE. Outcome and risk factors of ischemic heart disease in chronic uremia. Kidney Int 1996; 49: 1428-34.

14. Zoccali C, Benedetto FA, Mallamaci F, et al. Left ventricular mass monitoring in the follow-up of dialysis patients: prognostic value of left ventricular hypertrophy progression. Kidney Int 2004; 65: 1492-8.

15. Miyazato J, Horio T, Takiuchi S, et al. Left ventricular diastolic dysfunction in patients with chronic renal failure: impact of diabetes mellitus. Diabet Med 2005; 22: 730-6.

16. Agarwal S, Dangri P, Kalra OP, Rajpal S. Echocardiographic assessment of cardiac dysfunction in patients of chronic renal failure. JIACM 2003; 4: 296-303.

17. Hayashi SY, Rohani M, Lindholm B, et al. Left ventricular function in patients with chronic kidney disease evaluated by colour tissue Doppler velocity imaging. Nephrol Dial Transplant 2006; 21: 125-32.

18. McGregor E, Jardine AG, Murray LS, et al. Pre-operative echocardiographic abnormalities and adverse outcome following renal transplantation. Nephrol Dial Transplant 1998; 13: 1499-505.

19. Olechnowicz-Tietz S, Gluba A, Paradowska A, Banach M, Rysz J. The risk of atherosclerosis in patients with chronic kidney disease. Int Urol Nephrol 2013; 45: 1605-12.

20. Banach M, Serban C, Aronow WS, et al. Lipid, blood pressure and kidney update 2013. Int Urol Nephrol 2014; 46: 947-61.

21. Sharma R, Pellerin D, Gaze DC, et al. Mitral peak Doppler E-wave to peak mitral annulus velocity ratio is an accurate estimate of left ventricular filling pressure and predicts mortality in end-stage renal disease. J Am Soc Echocardiogr 2006; 19: 266-73.

22. Barberato SH, Pecoits-Filho R. Usefulness of left atrial volume for the differentiation of normal from pseudonormal diastolic function pattern in patients on hemodialysis. J Am Soc Echocardiogr 2007; 20: 359-65.

23. Yun KH, Kang DG, Kim KH, et al. The usefulness of colour M-mode Doppler echocardiographic indices in the 
assessment of left ventricular diastolic function. Korean Circ J 2004; 34: 1082-9.

24. Dokainish H, Zoghbi WA, Lakkis NM, et al. Optimal non-invasive assessment of left ventricular filling pressures: a comparison of tissue Doppler echocardiography and B-type natriuretic peptide in patients with pulmonary artery catheters. Circulation 2004; 109: 2432-9.

25. Pecková M, Charvat J, Schuck O, Hill M, Svab P, Horackova $M$. The association between left ventricular diastolic function and a mild-to-moderate decrease in glomerular filtration rate in patients with type 2 diabetes mellitus. J Int Med Res 2011; 39: 2178-86.

26. Levey AS, Astor BC, Stevens LA, et al. Chronic kidney disease, diabetes, and hypertension: what's in a name? Kidney Int 2010; 78: 19-22.

27. Innelli P, Sanchez R, Marra F, et al. The impact of aging on left ventricular longitudinal function in healthy subjects: a pulsed tissue Doppler study. Eur J Echocardiogr 2008; 9: 241-9.

28. Barberato SH, Mantilla DE, Misocami MA, et al. Effect of preload reduction by hemodialysis on left atrial volume and echocardiographic Doppler parameters in patients with end stage renal disease. Am J Cardiol 2004; 94: 1208-10.

29. Appleton CP, Hatle LK. The natural history of left ventricular filling abnormalities: assessment by two dimensional and Doppler echocardiography. Echocardiography 1992; 9: 437-57.

30. London GM. Cardiovascular disease in chronic renal failure: pathophysiologic aspects. Semin Dial 2003; 16: 85-94.

31. Crews DC, Plantinga LC, Miller ER 3rd, et al. Prevalence of chronic kidney disease in persons with undiagnosed or prehypertension in the United States. Hypertension 2010; 55: 1102-9.

32. Pecoits-Filho R, Barberato SH. Echocardiography in chronic kidney disease: diagnostic and prognostic implications. Nephron Clin Pract 2010; 114: c242-7.

33. Dougherty AH, Naccarelli GV, Gray EL, Hicks CH, Goldstein RA. Congestive heart failure with normal systolic function. Am J Cardiol 1984; 54: 778-82.

34. Soufer R, Wohlgelernter D, Vita NA, et al. Intact systolic left ventricular function in clinical congestive heart failure. Am J Cardiol 1985; 55: 1032-6.

35. Aronow WS. What should the optimal blood pressure goal be in patients with diabetes mellitus or chronic kidney disease? Arch Med Sci 2012; 8: 399-402.

36. Feola M, Lombardo E, Testa M, Avogadri E, Piccolo S, Vado A. Prognostic factors of mid-term clinical outcome in congestive heart failure patients discharged after acute decompensation. Arch Med Sci 2012; 8: 462-70.

37. Kunz K, Dimitrov Y, Muller S, Chantrel F, Hannedouche T. Uraemic cardiomyopathy. Nephrol Dial Transplant 1998; 13 (Suppl 4): 39-43

38. Franczyk-Skóra B, Gluba A, Banach M, Rozentryt $P$ Poloński L, Rysz J. Acute coronary syndromes in patients with chronic kidney disease. Curr Vasc Pharmacol 2013; 11: 758-67.

39. Franczyk-Skóra B, Gluba A, Banach M, Kozłowski D, Małyszko J, Rysz J. Prevention of sudden cardiac death in patients with chronic kidney disease. BMC Nephrol 2012; 13: 162

40. Foley RN, Parfrey PS, Harnett JD, Kent GM, Murray DC, Barre PE. The prognostic importance of left ventricular geometry in uremic cardiomyopathy. J Am Soc Nephrol 1995; 5: 2024-31
41. Foley RN, Parfrey PS, Harnett JD, et al. Clinical and echocardiographic disease in patients starting end-stage re nal disease therapy. Kidney Int 1995; 47: 186-92.

42. London GM, Pannier B, Guerin AP, et al. Alterations of left ventricular hypertrophy in and survival of patients receiving hemodialysis: follow-up of an interventional study. J Am Soc Nephrol 2001; 12: 2759-67.

43. Levin A, Singer J, Thompson CR, Ross H, Lewis M. Prevalent left ventricular hypertrophy in the predialysis population: identifying opportunities for intervention. Am J Kidney Dis 1996; 27: 347-54.

44. London GM, Parfrey PS. Cardiac disease in chronic uremia: pathogenesis. Adv Ren Replace Ther 1997; 4: 194-211.

45. Ori Y, Korzets A, Katz M, Perek Y, Zahavi I, Gafter U. Haemodialysis arteriovenous access: a prospective haemodynamic evaluation. Nephrol Dial Transplant 1996; 11: 94-7.

46. London GM, Guerin AP, Marchais SJ. Hemodynamic overload in end-stage renal disease patients. Semin Dial 1999; 12: 7-83.

47. Duran M, Unal A, Inanc MT, Esin F, Yilmaz Y, Ornek E. Effect of maintenance hemodialysis on diastolic left ventricular function in end-stage renal disease. Clinics 2010; 65: 979-84.

48. Rosello A, Torregrosa I, Solis MA. Study of diastolic function in peritoneal dialysis patients. Comparison between pulsed and Tissue Doppler. Nefrologia 2007; 27: 482-8.

49. Colantonio LD, Baber U, Banach M, et al. Contrasting cholesterol management guidelines for adults with CKD. J Am Soc Nephrol in press; doi: 10.1681/ ASN.2014040400.

50. Cicero AF, Reggi A, Parini A, Borghi C. Application of polyunsaturated fatty acids in internal medicine: beyond the established cardiovascular effects. Arch Med Sci 2012; 8: 784-93.

51. Banach M, Mikhailidis DP, Kjeldsen SE, Rysz J. Time for new indications for statins? Med Sci Monit 2009; 15: MS1-5. 